\title{
Variable Neighborhood Descent with Iterated Local Search for Routing and Wavelength Assignment
}

\author{
Alexandre X. Martins* Christophe Duhamel ${ }^{\dagger} \quad$ Philippe Mahey ${ }^{\ddagger}$ \\ Rodney R. Saldanha ${ }^{\S} \quad$ Mauricio C. de Souza ${ }^{\mathbb{N}}$
}

October 27, 2011

\begin{abstract}
In this work we treat the Routing and Wavelength Assignment (RWA) with focus on minimizing the number of wavelengths to route demand requests. Lightpaths are used to carry the traffic optically between origin-destination pairs. The RWA is subjected to wavelength continuity constraints, and a particular wavelength cannot be assigned to two different lightpaths sharing a common physical link. We develop a Variable Neighborhood Descent (VND) with Iterated Local Search (ILS) for the problem. In a VND phase we try to rearrange requests between subgraphs associated to subsets of a partition of the set of lightpath requests. In a feasible solution, lightpaths belonging to a subset can be routed with the same wavelength. Thus, the purpose is to eliminate one subset of the partition. When VND fails, we perform a ILS phase to disturb the requests distribution among the subsets of the partition. An iteration of the algorithm alternates between a VND phase and a ILS phase. We
\end{abstract}

*Programa de Pós-Graduação em Engenharia Elétrica, Universidade Federal de Minas Gerais, Av. Antônio Carlos, 6627, cep : 31270-901, Belo Horizonte, MG, Brasil, and Departamento de Ciências Exatas e Aplicadas, Universidade Federal de Ouro Preto, João Monlevade, MG, Brasil. e-mail : xmartins@decea.ufop.br

†Laboratoire LIMOS, CNRS-UMR6158, and ISIMA, Université Blaise Pascal, Campus des Cézeaux, BP 10125, 63173 Aubière CEDEX, France. e-mail : christophe.duhamel@isima.fr

${ }^{\ddagger}$ Laboratoire LIMOS, CNRS-UMR6158, and ISIMA, Université Blaise Pascal, Campus des Cézeaux, BP 10125, 63173 Aubière CEDEX, France. e-mail : philippe.mahey@isima.fr

$\S$ Departamento de Engenharia Elétrica, Universidade Federal de Minas Gerais, Av. Antônio Carlos, 6627, cep : 31270-901, Belo Horizonte, MG, Brasil. e-mail : rodney@cpdee.ufmg.br

`Departamento de Engenharia de Produção, Universidade Federal de Minas Gerais, Av. Antônio Carlos, 6627, cep : 31270-901, Belo Horizonte, MG, Brasil. e-mail : mauricio.souza@pq.cnpq.br

${ }$ corresponding author 
report computational experiments that show VND-ILS was able to improve results upon powerful methods proposed in the literature.

Keywords : Routing and assignment; Network design; Variable neighborhood descent; Local search

\section{Introduction}

Optical networks with wavelength division multiplexing (WDM) provide users with very large bandwidths. In all-optical networks a traffic demand is carried from source to destination through a lightpath, which is a sequence of fiber links carrying the traffic optically from end to end [5]. The wavelength continuity constraint implies that to a given lightpath a single wavelength must be assigned, i.e., a particular wavelength must be reserved to travel with the traffic on each link the lightpath traverses. Moreover, a particular wavelength cannot be assigned to two different lightpaths sharing a common physical link.

The Routing and Wavelength Assignment (RWA) problem deals with the routing and the assignment of wavelengths to lightpath requests between pairs of nodes. Given a set of lightpath requests, two variants of the RWA problem have been studied in the literature: max-RWA and min-RWA. In the former, the objective is to maximize the number lightpath requests that can be routed with a fixed number of wavelengths. In the latter, the objective is to minimize the number of wavelengths to route all the requests.

In this paper, we develop a Variable Neighborhood Descent (VND) with an Iterated Local Search (ILS) based perturbation for the min-RWA. Let $G=(V, E)$ be a digraph where $V$ is the set of nodes and $E$ is the set of bidirectional arcs. We denote by $\Gamma$ the set of lightpath requests where each $r \in \Gamma$ is defined by an origin and destination pair $\left(s_{r}, d_{r}\right) \in V \times V$. Note that we can have two different requests with the same origindestination pair and in this case if they are to be routed with the same wavelength, then they have to be routed through two arc disjoint paths in $G$. The problem is to find a minimal partition of $\Gamma$ in $W$ subsets such that the requests in each $\Gamma_{w}$, $w=1, \ldots, W$, can be routed through arc disjoint paths in $G$.

The paper is structured as follows. In the next section we discuss related works in the literature. In Section 3 we describe the VND-ILS heuristic for the min-RWA. Then, in Section 4 we report numerical results on some hardest benchmark instances from the literature. We end with concluding remarks and extensions for future work. 


\section{Related works}

Chlamtac et al. [4] have shown that the RWA problem is NP-Complete, and proposed, to our knowledge, the first greedy heuristics for the problem. Dutta and Rouskas [5] and Zang et al. [26] reviewed the literature on the RWA problem covering different approaches and variants developed in the 90's. For instance, Ramaswami and Sivarajan [23] derived upper bounds on the number of lightpath requests that can be routed, while in [24] they proposed to minimize the network congestion subjected to average delay constraints. Banerjee and Mukherjee [3] partitioned large RWA problems into several smaller subproblems to be solved independently and efficiently. Mukherjee et al. [19] proposed to alternate two phases: simulated annealing to construct a topology, and flow deviation to route the traffic. See also Kennington et al. [12] for the RWA problem in survivable WDM networks.

Mathematical formulations have been developed for the RWA problem, with particular emphasis on path-based ones. Ramaswami and Sivarajan [23] first modeled the RWA problem using a path-based formulation. Krishnaswamy and Sivarajan [13, 14] presented a mixed integer linear formulation which takes into account the maximum number of hops, among other logical and physical constraints, to minimize congestion. The authors developed heuristics based on rounding the solutions obtained by solving the respective LP-relaxation. Lee et al. [15] proposed a column generation algorithm to deal with the exponential number of variables associated to potential feasible paths. Jaumard et al. [9, 10], in comprehensive surveys, reviewed existing column generation formulations and proposed new ones. In particular, Jaumard et al. [11] developed improvements to make column generation methods efficient on the RWA problem. In their work, several instances were solved to optimality for the first time.

Some studies and tests have been done in order to design new or improved heuristics. The heuristics described below were developed for the min-RWA version of the problem. Manohar et el. [17] presented a greedy algorithm to do a partition of lightpath requests into subsets, each of which associated to a wavelength. Their algorithm explores techniques employed to solve the maximum edge disjoint paths problem. Noronha and Ribeiro [22] used a decomposition scheme in two distinct phases. In the first phase, a number of alternative routes are computed for each lightpath request. A conflict graph is built to exploit efficient heuristics for coloring problems. A route computed generates a node in the conflict graph, and an edge is set up between two nodes whose routes share a common link in the original graph. The set of nodes of the conflict graph is partitioned such that each subset contains the routes computed for a given lightpath request. Noronha and Ribeiro [22] proposed a tabu search for the partition coloring problem which is applied, in the second phase, over the conflict graph to assign wavelengths to routes. 
Skorin-Kapov [25] adapted ideas from bin packing heuristics to the min-RWA. For such purpose, she considered lightpath requests as items, and copies of the original graph as bins. The equivalent for the weight of an item is the number of links to route a lightpath. To say that a bin has not enough capacity to accommodate two items is equivalent to not being able to route two lightpath requests on a copy of the original graph without sharing a link. According to the bin packing analogy SkorinKapov [25] proposed four variants of best fit and first fit heuristics. In the numerical results reported in [25] the best fit decreasing (BFD) was the most successful among them. Noronha et al. [20] improved performance of best fit and first fit heuristics. To do this, the authors worked on data structures and implementation strategies, such as double linked adjacency lists and dynamically updating of shortest paths. Noronha et al. [20] introduced a broader set of testbed instances, and maximum running times were reduced to one quarter of those with a standard implementation of BFD (which was confirmed as the heuristic to perform best).

More recently, Noronha et al. [21] embedded BFD into a biased random-key genetic algorithm. The chromosomes are vectors of real numbers, denoted keys, in the interval $[0,1]$. The keys are used to bias a decoding heuristic in generating a feasible solution. Actually, each key is associated with a lightpath. Lightpaths are sorted in non-increasing order of the sum of their lengths and keys, and then BFD is applied. Computational experiments were conducted on the most studied instances and as well on the new benchmark ones introduced in [20]. The genetic algorithm improved upon results from heuristics previously proposed in the literature. Noronha et al. [21] reported that it reached solutions better than or similar to those found by a multistart variant of $\mathrm{BFD}$ in $23 \%$ less time on average, and reduced on average to half gaps provided by the decomposition scheme proposed in [22].

\section{VND-ILS heuristic for the min-RWA}

A feasible solution is characterized by a partition of $\Gamma$ in $W$ subsets along with arcs disjoints paths to route requests belonging to each $\Gamma_{w}, w=1, \ldots, W$, in $G$. Let us define by $F_{r} \subseteq E$ the arcs of the path used to route a request $r \in \Gamma$ in $G$. Each $\Gamma_{w} \subseteq \Gamma$ induces a subgraph $G_{w}=\left(V, E_{w}\right)$ of $G$ where $E_{w}=E-\cup_{r \in \Gamma_{w}} F_{r}$. In other words, $G_{w}$ contains the arcs not used to route requests belonging to $\Gamma_{w}$.

Given a feasible solution, in a VND phase we employ three kinds of moves trying to rearrange requests between the subgraphs associated to subsets of the partition in attempt to eliminate one of them. When VND fails, we perform a ILS-based perturbation phase to disturb the requests distribution among the subsets of the partition. Thus, an iteration of the algorithm alternates between a VND phase and a 
ILS-based perturbation phase.

\subsection{VND phase}

VND is a search heuristic proposed by Mladenović and Hansen [18] within the framework of variable neighborhood search methods, see Hansen et al. [6, 7, 8]. The VND works with $k_{\max }$ neighborhood structures $N_{k}, k=1, \ldots, k_{\max }$, designed for a specific problem. It starts with a given feasible solution as incumbent and sets $k=1$. If an improvement is obtained within neighborhood $N_{k}$, the method updates the new incumbent and sets $k=1$. Otherwise, it increases the value of $k$ and the next neighborhood is considered. The method stops when a local optimum for $N_{k_{\max }}$ is found.

We propose a VND algorithm with $k_{\max }=3$. Let $\Gamma_{\bar{w}}$ be a subset contained in the partition characterizing a feasible solution. We consider two alternatives to chose $\Gamma_{\bar{w}}$. In the first alternative, denoted by $\mathrm{VND}_{r}, \Gamma_{\bar{w}}$ is the subset of the partition with the least number of requests, i.e., $\bar{w}=\arg \min \left\{\left|\Gamma_{w}\right|: w=1, \ldots, W\right\}$. The idea is that it might be easier to empty by reallocating requests a set with a few of them. In the second one, denoted by $\mathrm{VND}_{e}, \Gamma_{\bar{w}}$ is the subset whose induced graph $G_{\bar{w}}$ has the greatest number of arcs, i.e., $\bar{w}=\arg \max \left\{\left|E_{w}\right|: w=1, \ldots, W\right\}$. If a fewer number of arcs were used to route requests belonging to $\Gamma_{\bar{w}}$ then it might be possible to find paths to route them in the subgraphs induced by the other sets of the partition. In both alternatives, the requests belonging to $\Gamma_{\bar{w}}$ form a list $L_{\bar{w}}$. The VND traverses $L_{\bar{w}}$ and tries with each request $r$ to perform moves within neighborhoods $N_{1}, N_{2}, N_{3}$ until it either succeeds to reallocate all requests belonging to $\Gamma_{\bar{w}}$ or fails to reallocate a request within $N_{3}$. In the former case, VND has emptied subset $\Gamma_{\bar{w}}$ of the partition, and consequently reduced the number of wavelengths to be used. In the latter case, the ILS-based perturbation phase is called. We remark that an improving move, if it happens, occurs only when the last remaining request of $\Gamma_{\bar{w}}$ is reallocated to another subset, while for $\left|\Gamma_{\bar{w}}\right| \geq 2$ performing a move does not reduce the objective function's value. When VND succeeds to empty $\Gamma_{\bar{w}}$, we update the remaining subsets of the partition with respect to the criteria to select the next subset to which VND is to be applied, according to the alternatives $\mathrm{VND}_{r}$ or $\mathrm{VND}_{e}$.

Suppose, traversing $L_{\bar{w}}$, the search is to consider a request $r$. A move in the first neighborhood $N_{1}$ tries to reallocate $r$ to another subset of $\Gamma$. If the neighborhood $N_{1}$ is not empty with respect to a partition and a request, then there exists $\Gamma_{w^{\prime}} \subset \Gamma$, $w^{\prime} \neq \bar{w}$, whose induced graph $G_{w^{\prime}}$ has a path between $s_{r}$ and $d_{r}$ (the request's origin and destination pair). In this case the search within $N_{1}$ stops when the first graph $G_{w^{\prime}}$ to accommodate $r$ is found, and $r$ is transferred from $\Gamma_{\bar{w}}$ to $\Gamma_{w^{\prime}}$. The search then continues to the next request in $L_{\bar{w}}$, or reduces a wavelength if $r$ was the last of $L_{\bar{w}}$. The list $L_{\bar{w}}$ is sorted in non-increasing order of shortest path lengths in $G$ 
between origin and destination nodes. The motivation behind is to try to route in the subgraphs induced by the other sets first the requests using more arcs, and leave the requests using fewer arcs to try after since when a path is found to route a request in a subgraph its arcs are removed. If $N_{1}$ is empty, VND proceeds to neighborhood $N_{2}$ with the same request $r$ under analysis.

The rationale in the second neighborhood is try first to make room for $r$ in a graph $G_{w^{\prime}}$ by transferring as many requests as possible from $\Gamma_{w^{\prime}}$ to other subsets of $\Gamma$ not equal to $\Gamma_{\bar{w}}$. This is done analogously to the search in neighborhood $N_{1}$, i.e., we traverse $L_{w^{\prime}}$ and try, for each request, to find a subset $\Gamma_{\hat{w}}, \hat{w} \neq w^{\prime}$ and $\hat{w} \neq \bar{w}$, such there is a path to route the request in $G_{\hat{w}}$. If we are able to unload at least one request from $\Gamma_{w^{\prime}}$, then we can try to allocate $r$ to it. Upon success, the search resumes from the next request in $L_{\bar{w}}$, if any, within neighborhood $N_{1}$. Otherwise, VND proceeds to neighborhood $N_{3}$ still with the same request $r$ under analysis. Assume that the subsets of the partition were built in a given order, $w=1, \ldots, W$. At each new search within $N_{2}$ we take only one subset of the partition, according to the order, to try to make room for $r$ in it. That is, if we fail, during a search within $N_{2}$, to make room in a graph $G_{w^{\prime}}$ for request $r$, we do not try to perform a move considering another graph but proceeds to neighborhood $N_{3}$. Then, the next time we proceed to $N_{2}$ the graph to be considered will be $G_{w^{\prime}+1}$ (assuming $\bar{w} \neq w^{\prime}+1$ ).

A move in neighborhood $N_{3}$ seeks to swap request $r$ with another request belonging to a subset of $\Gamma$ different from $\Gamma_{\bar{w}}$. Given a request $u$, let $\min _{s p l}(u)$ be the number of arcs of the shortest path between $s_{u}$ and $d_{u}$ in $G$. The search considers requests with $\min _{s p l}$ smaller than $\min _{s p l}(r)$, sorted in non-decreasing order of $\min _{s p l}$. The choice to consider only requests whose shortest paths in $G$ have fewer arcs than $r$ carries the idea that it might be easier to route such a request in other subgraphs of the partition when the search resumes from $N_{1}$. Thus, if a request $r^{\prime} \in \Gamma_{w^{\prime}}$, such that $r$ can be routed in the subgraph induced in $G$ by $\Gamma_{w^{\prime}}-\left\{r^{\prime}\right\}$, and $r^{\prime}$ in the one by $\Gamma_{\bar{w}}-\{r\}$, is found, then a swap between $r$ and $r^{\prime}$ is performed. After a successful move in $N_{3}$ we have $r^{\prime} \in \Gamma_{\bar{w}}$ and $r \in \Gamma_{w^{\prime}}$. In this case, the search resumes by trying to transfer $r^{\prime}$ from $\Gamma_{\bar{w}}$ to another subset of the partition with a move in $N_{1}$. Note that a move in $N_{3}$ does not reduce $\left|\Gamma_{\bar{w}}\right|$, but since it swaps $r$ with $r^{\prime}$, and $\min _{s p l}\left(r^{\prime}\right)<\min _{s p l}(r)$, it may allow a reallocation of $r^{\prime}$ with a move in $N_{1}$ in the sequel. If it is not possible within $N_{3}$ to swap $r$ with any other request having a smaller $\min _{\text {spl }}$, the search calls a ILS-based perturbation phase. This means that VND failed to empty $\Gamma_{\bar{w}}$, and it is not subsequently applied to another subset of the partition. Instead, a ILS-based perturbation phase is applied to disturb the current solution before another trial of VND.

Figure 1 illustrates searching moves within each neighborhood of VND. The idea in part $1(\mathrm{a})$ is to move request $r$ from $\Gamma_{\bar{w}}$ to a different subset $\Gamma_{i}, i=1, \ldots, W, i \neq \bar{w}$, 
as long as $r$ can be routed in $G_{i}$. Part 1(b) shows the attempt to accommodate $r$ in $\Gamma_{w^{\prime}}$ by reallocating as much requests from $\Gamma_{w^{\prime}}$ as possible. Part 1(c) represents swap moves.

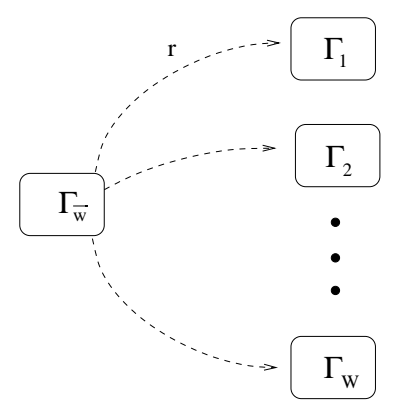

(a) $N_{1}$

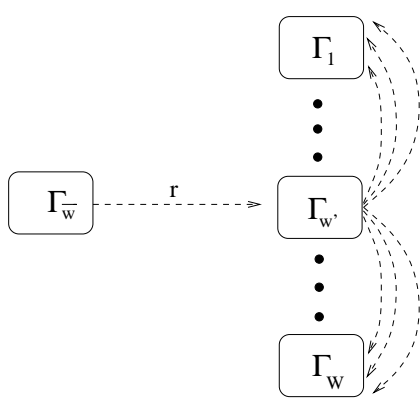

(b) $N_{2}$

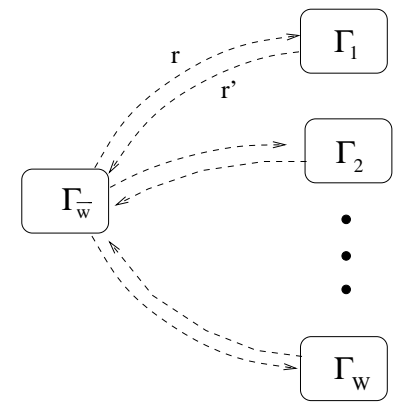

(c) $N_{3}$

Figure 1: Searching moves within neighborhoods $N_{1}, N_{2}$, and $N_{3}$ of VND.

\subsection{ILS-based perturbation phase}

ILS employs perturbation techniques to escape from a current local optima. Let $s^{*}$ be a current solution, and let initially $s$ be equal to $s^{*}$, then ILS's iterative step has three components: (i) a local search applied to $s$ generating $s^{\prime}$; (ii) an acceptance criterion to either update $s^{*}$ to $s^{\prime}$ or not; and (iii) a perturbation technique applied to $s^{*}$ generating a new solution $s$. The overall best solution is returned after a number of iterations. The reader is referred to Lourenço et al. [16] for a broader discussion on ILS features.

We exploit the idea of applying a perturbation technique, in the manner of ILS, after a solution had been obtained by the VND. Suppose the perturbation is applied when VND failed to swap a request $r \in \Gamma_{\bar{w}}$ within $N_{3}$. Note that at this point VND might have successfully performed moves when traversing the requests' list $L_{\bar{w}}$. We do not undo these moves and consequently, when perturbation is applied, the subsets of $\Gamma$ characterizing the partition may have other elements than those at the beginning of the iteration. The perturbation relies upon an assignment problem built to rearrange requests among the subsets partitioning $\Gamma-\Gamma_{\bar{w}}$. We pull out a randomly chosen request $r_{i}$ from each subset $\Gamma_{i}, \Gamma_{i} \subseteq \Gamma-\Gamma_{\bar{w}}$, of the partition. Let $G_{i}^{a}=\left(V, E_{i}^{a}\right)$ be the subgraph of $G$ where $E_{i}^{a}=E_{i} \cup F_{r_{i}}$ (the arcs used to route request $r_{i}$ are reactivated in $G_{i}^{a}$ ). The perturbation is done by finding the best way to assign $W-1$ requests chosen to $W-1$ subsets partitioning $\Gamma-\Gamma_{\bar{w}}$. The cost of assigning request $r_{i} \in \Gamma_{i}$ to subset $\Gamma_{j}$ is given as follows:

- $c_{r_{i} j}=2$, if $j=i$; 
- $c_{r_{i} j}=\infty$, if there is no path to route request $r_{i}$ in the graph $G_{j}^{a}$;

- $c_{r_{i} j}=1-\frac{\min _{s p l}\left(r_{i}\right)}{\min _{s p l}\left(r_{i}\right)\left(G_{j}^{a}\right)}-\max \left(\min _{s p l}\left(r_{i}\right)\left(G_{i}^{a}\right)-\min _{s p l}\left(r_{i}\right)\left(G_{j}^{a}\right), 0\right)$, otherwise;

where $\min _{\text {spl }}\left(r_{i}\right)\left(G^{\prime}\right)$ is the number of arcs of the shortest path between $s_{r_{i}}$ and $d_{r_{i}}$ in graph $G^{\prime}$ (we remember that $\min _{s p l}\left(r_{i}\right)$ is the number of arcs of the shortest path in the original graph $G$ ). The assignment problem results in a feasible partition of $\Gamma-\Gamma_{\bar{w}}$ because a solution that returns each $r_{i}$ to its original subset $\Gamma_{i}$ is always feasible and possess a cost of $2(W-1)$, which is smaller than the cost of any solution that assigns a request $r_{i}$ to a subset $\Gamma_{j}$ where there is no path to route $r_{i}$ in $G_{j}^{a}$. If the solution of the assignment problem has cost smaller than $2(W-1)$, then at least two requests were assigned to subsets other than their original ones while preserving feasibility in terms of arc disjoint paths to route requests of each subset. Thus, after solving the assignment problem, we check whether the allocation of requests to partition subsets has changed. If so, the algorithm resumes the VND by trying a move with $r \in \Gamma_{\bar{w}}$ within $N_{1}$. Otherwise, a perturbation step is done again. Figure 2 shows an example where $\Gamma$ is partitioned in five subsets. Suppose VND failed to swap a request $r \in \Gamma_{4}$ within $N_{3}$. Then, for each $i, i \in\{1,2,3,5\}$, a request $r_{i}$ is randomly chosen and the assignment problem built. Note the graph $G_{4}(\bar{w}=4)$ does not take part on the assignment.

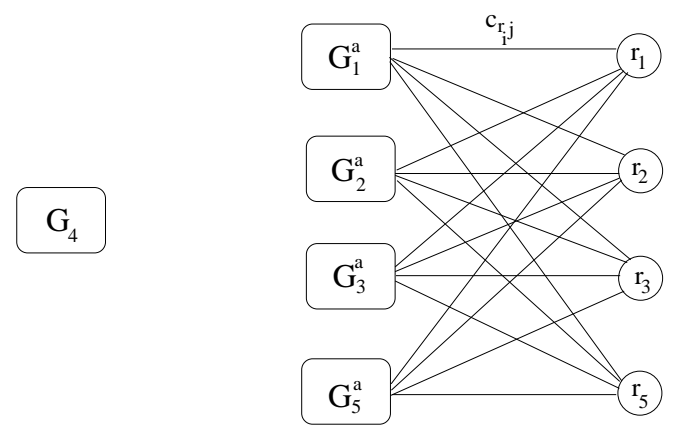

Figure 2: ILS-based perturbation by means of an assignment problem.

We propose two variants for choosing a request $r_{i}$ from subset $\Gamma_{i}$ to build the assignment problem used to guide the perturbation. In the first one, denoted ILS $_{p}$, all requests belonging to $\Gamma_{i}$ have the same probability to be chosen. In the second one, denoted $\mathrm{ILS}_{5 p}$, requests whose paths pass through the origin $s_{r_{i}}$ or the destination $d_{r_{i}}$ of the request $r \in \Gamma_{\bar{w}}$ for which VND failed to perform a move within $N_{3}$ have higher probability of being chosen. In fact, if the path used to route request $r_{i} \in \Gamma_{i}$ in $G$ has an outgoing (resp. incoming) arc from $s_{r_{i}}$ (resp. to $d_{r_{i}}$ ), then $r_{i}$ has five times more probability of being chosen. 


\section{Computational results}

The computational experiments were structured into three comparative settings: comparison among combinations of VND-ILS strategies, evaluation of the effectiveness of the VND-ILS with respect to a multistart heuristic and robustness to initial solution, and comparison with the strongest metaheuristic in the literature. For such purpose, we used benchmark instances from the literature - the most studied realistic instances, and sets $Y$ and $Z$ introduced by Noronha et al. [20]. The realistic instances are available on the web ${ }^{1}$ or were provided along with sets $Y$ and $Z$ by Noronha ${ }^{2}$. Instances $Y$ and $Z$ are the most difficult ones. Set $Y$ is formed by randomly generated instances with 100 nodes and different values for the probabilities of a link and a request between a pair of nodes. Set $Z$ is formed by instances with 100 nodes on a grid embedded on a torus where each node is connected only to its nearest four nodes, and different values for the probability of a request between a pair of nodes. Computational experiments were carried out on a Core 2 Duo with $1.97 \mathrm{GHz}$ and $4 \mathrm{~GB}$ of RAM, running MS Windows XP, and the proposed algorithms were coded on $\mathrm{C}++$.

Table 1 presents average results to decide among VND-ILS alternatives the one that seems to work better. The first column lists the group of instances. The group Realistic has 26 instances, and each of the $Z$ and $Y$ groups has 5 and 25 instances, respectively. Then, for each combination VND-ILS we report average deviation gaps for 5 runs of the heuristic on each instance, each run limited to 5 minutes. The gaps are calculated as the difference in percentage between the upper bound UB obtained by the heuristic and the lower bound LB computed according to Jaumard et al. [9, 11] and also used in the study conducted by Noronha et al. [20, 21], i.e., (UB-LB)/LB. It can be seen that if the VND strategy is fixed, ILS $_{5 p}$ leads consistently to better results. It shows that giving more chance, in the ILS-based perturbation, to requests passing through the origin or destination nodes of the request blocked during the precedent VND phase is more effective in rearranging wavelengths to another VND trial. The different VND strategies have similar behavior, with $\mathrm{VND}_{e}$ obtaining slightly better results. Thus, we present in the sequel results comparing the $\mathrm{VND}_{e}-\mathrm{ILS}_{5 p}$ with the powerful methods in the literature.

The second comparison setting aims to see whether VND and ILS are effective to improve results regarding a multistart variant of what is considered the best constructive heuristic in the literature, and on the other hand evaluate the robustness of the method with respect to initial solution. The initial solution for $\mathrm{VND}_{e}-\mathrm{ILS}_{5 p}$ is given by one run of the BFD heuristic proposed by Skorin-Kapov [25]. Thus, we compare $\mathrm{VND}_{e}-\mathrm{ILS}_{5 p}$ to a multistart variant of BFD where requests whose shortest

\footnotetext{
${ }^{1}$ http://dag.cs.uni-kl.de/research/rwa/

${ }^{2}$ personal communication
} 
paths in $G$ have the same length are randomly ordered. We then apply solely $\mathrm{VND}_{e}$ to the initial solution. Finally, we apply $\mathrm{VND}_{e}-\mathrm{ILS}_{5 p}$ starting from a different initial solution which is obtained with the edge disjoint path (EDP) heuristic proposed by Manohar et al. [17].

Table 2 presents results of the four methods - Multistart-BDF, BFD-VND, $\mathrm{VND}_{e^{-}}$ $\mathrm{ILS}_{5 p}, \mathrm{EDP}-\mathrm{VND}_{e}-\mathrm{ILS}_{5 p}$ - on a set of realistic instances. For each method we performed again 5 runs on each instance, each of them limited to 5 minutes. The first column presents the instance's identification, and from the second to the fourth column the corresponding number of nodes, number of arcs, and number of lightpath requests. For the instances marked with $\left({ }^{*}\right)$ we randomly generated asymmetric requests. Then, in the next columns, we report for each method the minimum number of wavelengths obtained on 5 runs, that is the best solution found, and the average gap. The last column presents the lower bounds. It has seen in Table 1 that realistic instances are easier to solve than the ones in sets $Y$ and $Z$. Nevertheless, Multistart$\mathrm{BFD}$ and $\mathrm{BFD}-\mathrm{VND}_{e}$ were not able to find optimal solutions for all of them, whereas $\mathrm{VND}_{e}-\mathrm{ILS}_{5 p}$ and EDP-VND -ILS $_{5 p}$ did. We see from Table 2 that BFD-VND $\mathrm{Im}$ proved results upon Multistart-BFD reducing the overall average gap from $3.27 \%$ to $2.50 \%$, though BFD-VND $e_{e}$ still shows some high average gaps of $20 \%$ and $15.37 \%$. But when $\mathrm{ILS}_{5 p}$ was coupled, the method $\mathrm{VND}_{e}-\mathrm{ILS}_{5 p}$ closed all gaps independently of the initial solution.

Tables 3 to 5 present results on the set $Y$ of instances [20, 21]. These 100 node instances are characterized by the probabilities that there is a pair of direct arcs between a pair of nodes, which are 0.03, 0.04, 0.05, and that there is a request between a pair of nodes, which are $0.2,0.4,0.6,0.8,1$. There are 5 instances for each combination of probabilities. For example, instance y.3.60.4, has probability of 0.03 for arcs and 0.6 for requests, and it is the 4 th instance with this combination. Results are reported analogously to those in Table 2, and again 5 runs limited to 5 minutes were performed for each instance. Comparing the four methods, they follow the general tendency observed in Table 2. The VND solely produces only slightly reductions on average gaps, and both versions employing ILS-based perturbation lead to significant smaller gaps than Multistart-BFD. We note that $\mathrm{VND}_{e}-\mathrm{ILS}_{5 p}$ was able to improve gaps on all the 42 out of 75 instances for which Multistart-BFD was not able to find the optimum. Moreover, EDP-VND $e^{-\mathrm{ILS}_{5 p}}$, starting from a different initial solution, was able to obtain smaller gaps than Multistart-BFD on 35 out of these 42 instances. The use of BFD as initial solution yields however better results, since for these harder

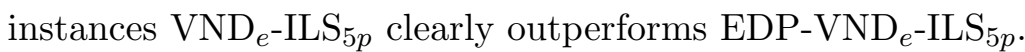

Table 6 presents results on the set $Z$ of instances [20,21]. These instances have approximately 100 nodes which are the vertices on grids of dimensions $10 \times 10,8 \times 13$, $6 \times 7,5 \times 20,4 \times 25$, with probability of $0.2,0.4,0.6,0.8,1$ to have a request between 
a pair of nodes. For example, instance z. $8 \times 13.80$, is a $8 \times 13$ grid with probability of 0.8 for requests. Results for 5 runs limited to 5 minutes for each method are reported in the same manner. Although gaps of $20 \%$ are not observed as in some cases of set $Y$, these instances seem to be more difficult because Multistart-BFD were not able to match lower bounds. And in this case $\mathrm{VND}_{e}-\mathrm{ILS}_{5 p}$ was able to obtain better gaps than Multistart-BFD for all 25 instances in set $Z$. Besides, $\mathrm{VND}_{e}-\mathrm{ILS}_{5 p}$ was able to find the optimum for 6 instances. As it can be seen from Table 6, the proposed approach of

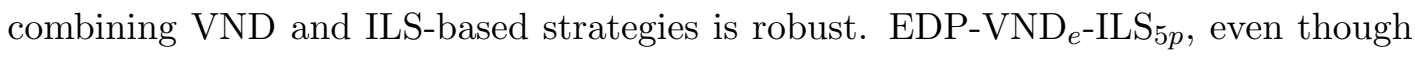
not using BFD to generate initial solutions, found better gaps than Multistart-BFD for 14 instances.

The third setting is intended to compare $\mathrm{VND}_{e}-\mathrm{ILS}_{5 p}$ with the genetic algorithm GA-RWA proposed by Noronha et al. [21] which recently improved the state-of-the-art algorithms in the literature. Noronha et al. [21] selected a subset of 30 instances as the hardest ones, and their results showed that GA-RWA was able to improve average gaps on 29 of them. Table 7 presents a comparison between $\mathrm{VND}_{e}-\mathrm{ILS}_{5 p}$ and GARWA on these 30 instances. Results of GA-RWA were obtained with a limit of 10 minutes on a Pentium IV with $3.4 \mathrm{GHz}$, and those of $\mathrm{VND}_{e}-\mathrm{ILS}_{5 p}$ with a limit of 5 minutes as an attempt to take into account the processor difference. The first column presents the instance. Then, the second and third (resp. the fourth and fifth) columns present the best solution and the average gap for 5 runs obtained by GA-RWA (resp. by $\left.\mathrm{VND}_{e}-\mathrm{ILS}_{5 p}\right)$. The last column presents the lower bound. Table 7 shows quite competitive results regarding the state-of-the-art in the literature. $\mathrm{VND}_{e}-\mathrm{ILS}_{5 p}$ found better solutions than GA-RWA in 26 out of 30 instances, and equal solutions for the other 4. Besides, $\mathrm{VND}_{e}-\mathrm{ILS}_{5 p}$ was able to find 6 optima for instances that were still open. In terms of time consuming, on average, the time spent on neighborhoods $N_{1}$ and $N_{2}$ is about $5 \%$, on neighborhood $N_{3}$ is about $25 \%$, and the most time consuming phase is the ILS-based perturbation with $70 \%$ of the CPU time.

We now proceed to detailed analysis on the behavior of algorithm $\mathrm{VND}_{e}-\mathrm{ILS}_{5 p}$. The first analysis is an attempt to learn more about the performance of $\mathrm{VND}_{e}-\mathrm{ILS}_{5 p}$ if it should run on less time than 5 minutes. For such purpose, we make use of the timeto-target (TTT) plots proposed by Aiex et al. [1, 2]. The hypothesis behind is that CPU times fit a two parameter exponential distribution. Thus, for a given problem instance, TTT plots display on the ordinate axis the probability for the heuristic to obtain a solution as good as a given target value within a running time in seconds, shown on the abscissa axis. We had chosen 8 instances from Table 7 to do this. For each instance we ran $\mathrm{VND}_{e}-\mathrm{ILS}_{5 p} 200$ times with different seeds until the algorithm reaches the target value. Figures 3 to 6 show the TTT plots generated with 4 instances for which an average gap of $0 \%$ is reached - ATT, NSF.12, y.4.20.4, y.5.100.2. For these instances we set the target value to the optima. On the other hand, Figures 7 
to 10 show the TTT plots generated with the instances for which a high average gap is observed - y.4.80.1, y.4.100.1, Z.10x10.60, Z.10x10.80. In these cases, we set the target value to one wavelength less than the best solution found by GA-RWA. TTT plots indicate that $\mathrm{VND}_{e}-\mathrm{ILS}_{5 p}$ is likely to obtain high quality solutions in a short time. As it can be seen, on instances tested, $\mathrm{VND}_{e}-\mathrm{ILS}_{5 p}$ has high probability to find very good solutions (optimum or better than the best known) in less than 2 minutes, except for instance y.5.100.2, for which a little more time is needed.

The second analysis tries to bring some insight on which neighborhood brings the best gain. We performed experiments with reduced versions of VND using, along with ILS-based perturbation, neighborhoods $N_{1}$ and $N_{2}$ separately and in combination with $N_{3}$. Neighborhood $N_{3}$ is not tested separately since alone it cannot reduce the number of wavelengths. Table 8 shows numerical results for the reduced versions of VND. The first column presents the instance. Then, the subsequent pairs of columns present the best solution and the average gap for each reduced version of VND. As before, it is reported for each proposed algorithm the results of 5 runs with a time limit of 5 minutes each. The last column presents the lower bound. We remark that although $N_{3}$ alone cannot be used to improve solutions, the reduced versions of VND using it in combination with $N_{1}$ or $N_{2}$ lead to the better results. So, a move in neighborhood $N_{3}$ is an important instrument to rearrange requests to further improving moves. It is also worth noting that neighborhood $N_{2}$ alone is not effective, but in combination with $N_{3}$ becomes the most successful reduced version of VND.

\section{Conclusions}

We propose an algorithm for the RWA problem that alternates between a VND phase and a ILS-based perturbation phase. In the VND phase we explore three neighborhoods by trying three kinds of moves. The purpose is to reduce one wavelength. The ILS-based phase is called to introduce a perturbation in the current partition of the lightpath requests' set. The perturbation itself does not improve a solution, but it leaves a rearrangement of lightpaths among subsets of the partition that may yield further improvements with another trial of VND. Even though better results were found taking BFD as constructive heuristic, VND-ILS is quite robust with respect to the initial solution and clearly outperforms a multistart variant of BFD. Computation experiments were conducted on the hardest benchmark instances, and significant improvements upon better upper bounds from the literature were achieved. An interesting research direction is to adapt the methods to deal with possibilities of traffic-grooming. 


\section{Acknowledgements}

The authors wish to thank the two anonymous referees for helpful suggestions in improving this paper. The authors from Brazilian institutions were partially supported by CAPES, CNPq, and FAPEMIG, Brazil.

\section{References}

[1] R.M. Aiex, M.G.C. Resende and C.C. Ribeiro, "Probability distribution of solution time in GRASP: an experimental investigation", Journal of Heuristics 8 (2002), 343-373.

[2] R.M. Aiex, M.G.C. Resende and C.C. Ribeiro, "TTT plots: a perl program to create time-to-target plots", Optimization Letters 1 (2007), 355-366.

[3] D. Banerjee and B. Mukherjee, "A practical approach for routing and wavelength assignment in large wavelength-routed optical networks", IEEE Journal on Selected Areas in Communications 14 (1996), 903-908.

[4] I. Chlamtac, A. Ganz, and G. Karmi, "Lightpath communications: An approach to high bandwidth optical WAN's", IEEE Transactions on Communications 40 (1992), 1171-1182.

[5] R. Dutta and G.N. Rouskas, "A survey of virtual topology design algorithms for wavelength routed optical networks", Optical Networks Magazine 1 (2000), $73-89$.

[6] P. Hansen, N. Mladenović "Variable neighborhood search", In: F. Glover, G. Kochenberger (Eds.), Handbook of Metaheuristics, Kluwer, 145-184, 2003.

[7] P. Hansen, N. Mladenović, J.A. Moreno Pérez, "Variable neighborhood search: methods and applications", 4OR A Quarterly Journal of Operations Research 6 (2008), 319-360.

[8] P. Hansen, N. Mladenović , J.A. Moreno Pérez, "Variable neighbourhood search: methods and applications", Annals of Operations Research 175 (2010), 367-407.

[9] B. Jaumard, C. Meyer, B. Thiongane, "ILP formulations for the RWA problem for symmetrical systems", In: P. Pardalos, M. Resende (Eds.), Handbook for Optimization in Telecommunications, Kluwer, 637-678, 2006.

[10] B. Jaumard, C. Meyer, B. Thiongane, "Comparison of ILP formulations for the RWA problem", Optical Switching and Networking 4 (2007), 157-172.

[11] B. Jaumard, C. Meyer, B. Thiongane, "On column generation formulations for the RWA problem", Discrete Applied Mathematics 157 (2009), 1291-1308. 
[12] J. Kennington, E. Olinick, A. Ortynski, and G. Spiride, "Wavelength routing and assignment in a survivable WDM mesh network", Operations Research 51 (2003), $67-79$.

[13] R.M. Krishnaswamy and K.N. Sivarajan, "Algorithms for routing and wavelength assignment based on solutions of LP-relaxations", IEEE Communications Letters 5 (2001), 435-437.

[14] R.M. Krishnaswamy and K.N. Sivarajan, "Design of logical topologies: A linear formulation for wavelength-routed optical networks with no wavelength changers", IEEE/ACM Transactions on Networking 9 (2001), 186-198.

[15] K. Lee, K.C. Kang, T. Lee, S. Park, "An optimization approach to routing and wavelength assignment in WDM all-optical mesh networks without wavelength conversion", ETRI Journal 24 (2002), 131-141.

[16] H.R. Lourenço, O.C. Martin, T. Stützle, "Iterated local search", In: P. Glover, G. Kochenberger (Eds.), Hanbook of Metaheuristics, 321-353, Springer, 2003.

[17] P. Manohar, D. Manjunath, R.K. Shevgaonkar, "Routing and wavelengths assignment in optical networks from edge disjoint path algorithms", IEEE Communication Letters 6 (2002), 211-213.

[18] N. Mladenović, P. Hansen, "Variable neighbourhood search", Computers $\& 5$ Operations Research 24 (1997), 1097-1100.

[19] B. Mukherjee, D. Banerjee, S. Ramamurthy, and A. Mukherjee, "Some principles for designing a wide-area WDM optical network", IEEE/ACM Transactions on Networking 4 (1996), 684-696.

[20] T. Noronha, M.G.C. Resende, C.C. Ribeiro, "Efficient implementations of heuristics for routing and wavelength assignment", In: C.C. McGeoch (Ed.), Proceedings of the 7th International Workshop on Experimental Algorithms, Lecture Notes in Computer Science 5038 (2008), 169-180.

[21] T. Noronha, M.G.C. Resende, C.C. Ribeiro, "A biased random-key genetic algorithm for routing and wavelength assignment", Journal of Global Optimization, DOI 10.1007/s10898-010-9608-7.

$[22]$ T. Noronha, C.C. Ribeiro, "Routing and wavelength assignment by partition colouring", European Journal of Operational Research 171 (2006), 797-810.

[23] R. Ramaswami and K.N. Sivarajan, "Routing and wavelength assignment in alloptical networks", IEEE/ACM Transactions on Networking 3 (1995), 489-500.

[24] R. Ramaswami and K.N. Sivarajan, "Design of logical topologies for wavelengthrouted optical networks", IEEE Journal on Selected Areas in Communications 14 (1996), 840-851. 
[25] N. Skorin-Kapov, "Routing and wavelength assignment in optical networks using bin packing based algorithms", European Journal of Operational Research 177 (2007), 1167-1179.

[26] H. Zang, J.P. Jue, and B. Mukherjee, "A review of routing and wavelength assignment approaches for wavelength-routed optical WDM networks", Optical Networks Magazine 1 (2000), 47-60.

\begin{tabular}{lcccc} 
Set & $\mathrm{VND}_{e}-\mathrm{ILS}_{p}$ & $\mathrm{VND}_{r}-\mathrm{ILS}_{p}$ & $\mathrm{VND}_{e}-\mathrm{ILS}_{5 p}$ & $\mathrm{VND}_{r}-\mathrm{ILS}_{5 p}$ \\
\hline Realistic & 0.00 & 0.00 & 0.00 & 0.00 \\
Z.20 & 2.72 & 2.57 & 2.36 & 2.39 \\
Z.40 & 3.27 & 3.15 & 2.75 & 2.78 \\
Z.60 & 3.11 & 3.08 & 2.55 & 2.62 \\
Z.80 & 2.66 & 2.63 & 2.41 & 2.40 \\
Z.100 & 4.15 & 4.15 & 3.94 & 3.88 \\
Y.3 & 4.25 & 4.20 & 3.66 & 3.75 \\
Y.4 & 6.62 & 6.60 & 6.37 & 6.39 \\
Y.5 & 1.79 & 1.66 & 1.30 & 1.33 \\
\hline Average & 3.17 & 3.12 & 2.82 & 2.84
\end{tabular}

Table 1: Deviation gaps in percentage for combinations of VND-ILS strategies. 


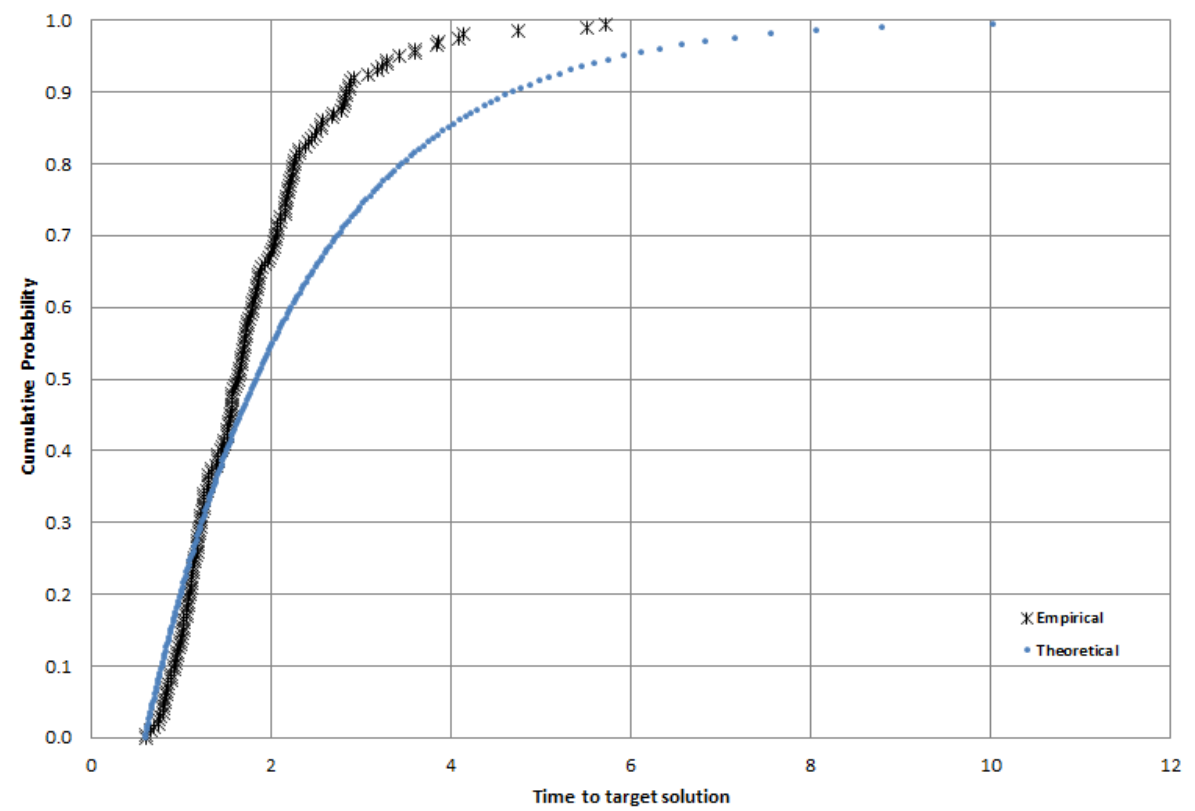

Figure 3: TTT plots produced for instance ATT - target value equal to 20.

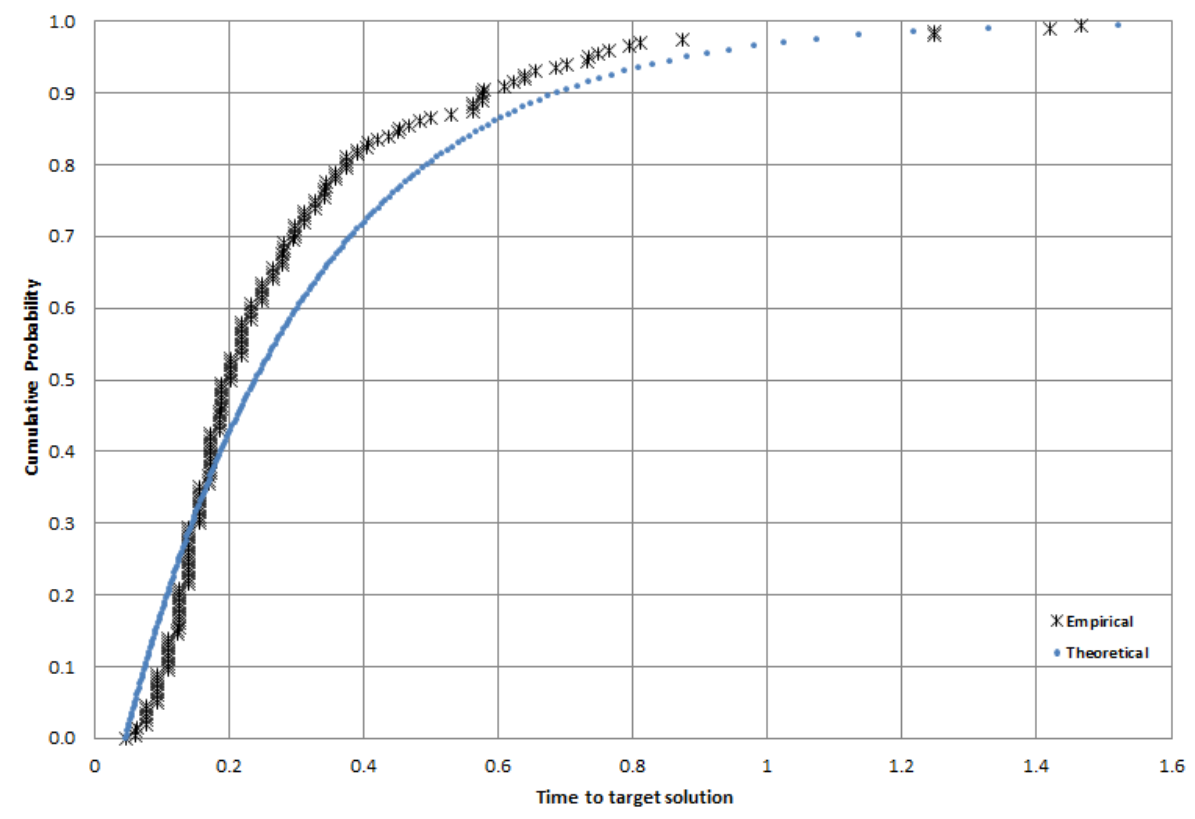

Figure 4: TTT plots produced for instance NSF.12 - target value equal to 38. 


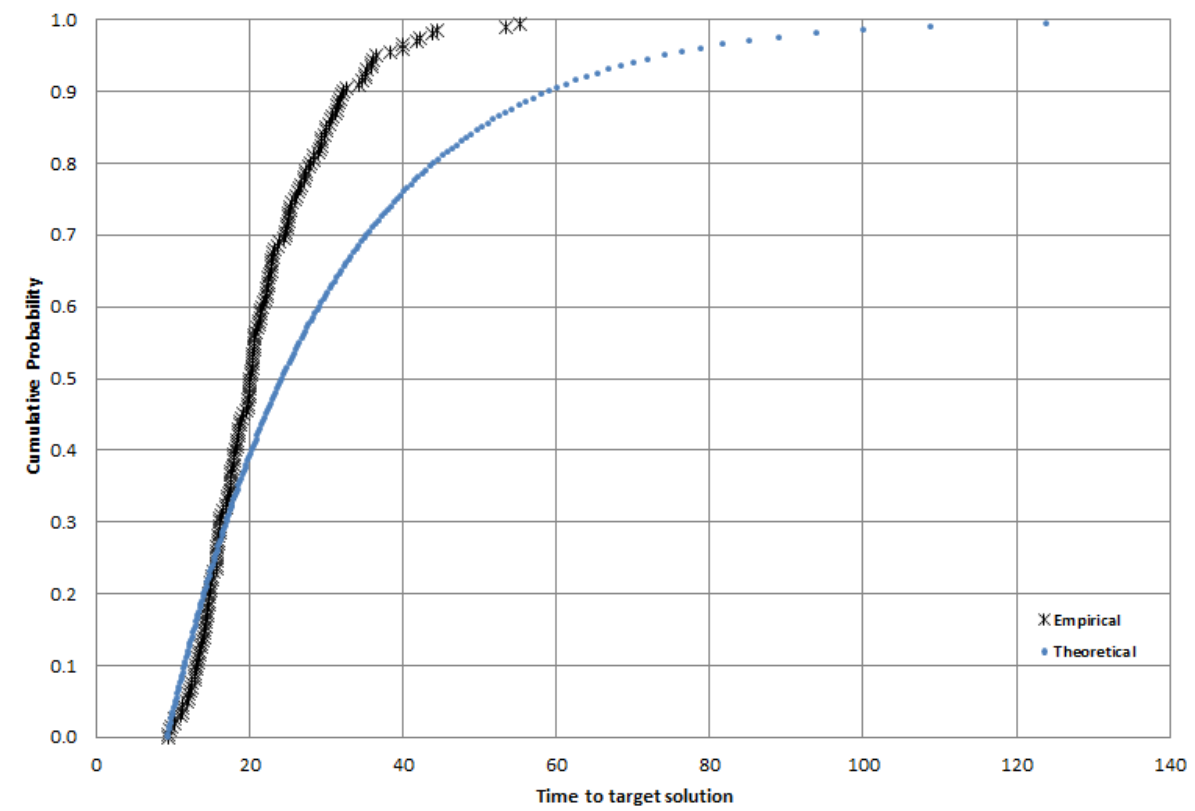

Figure 5: TTT plots produced for instance y.4.20.4 - target value equal to 19.

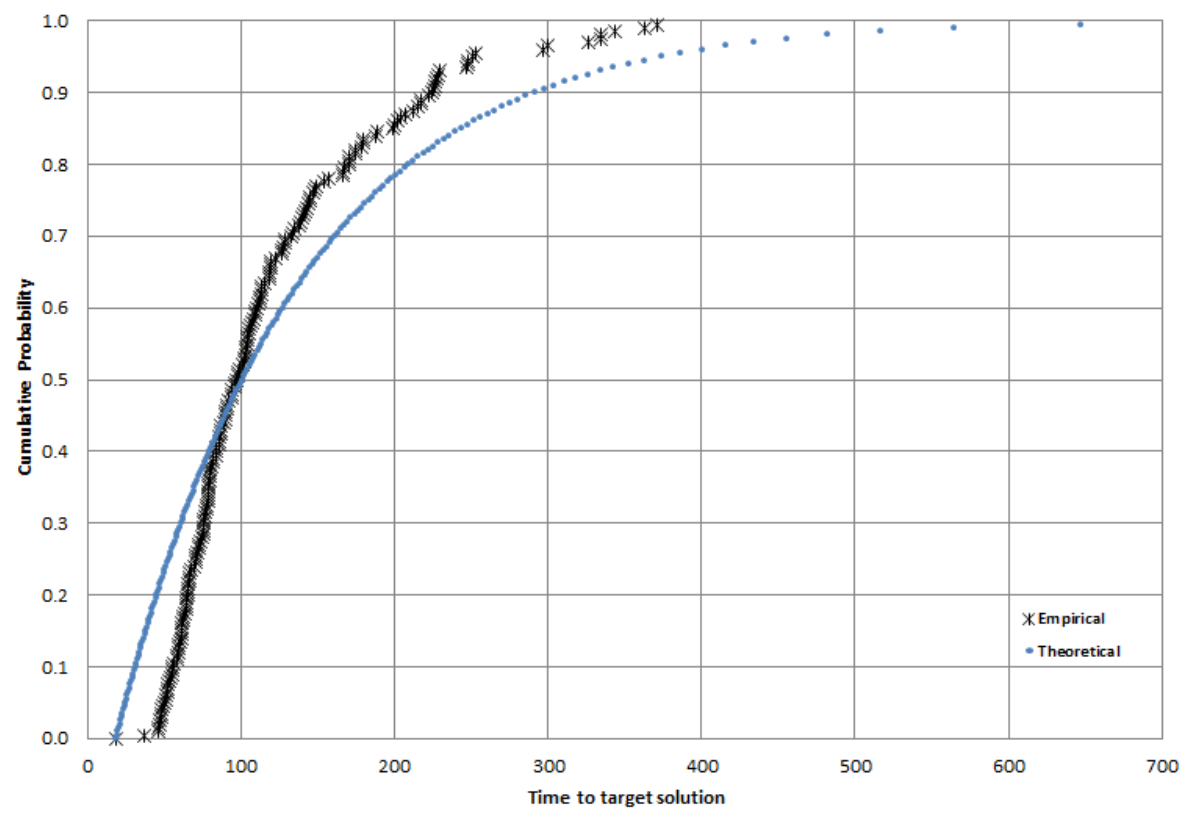

Figure 6: TTT plots produced for instance y.5.100.2 - target value equal to 73. 


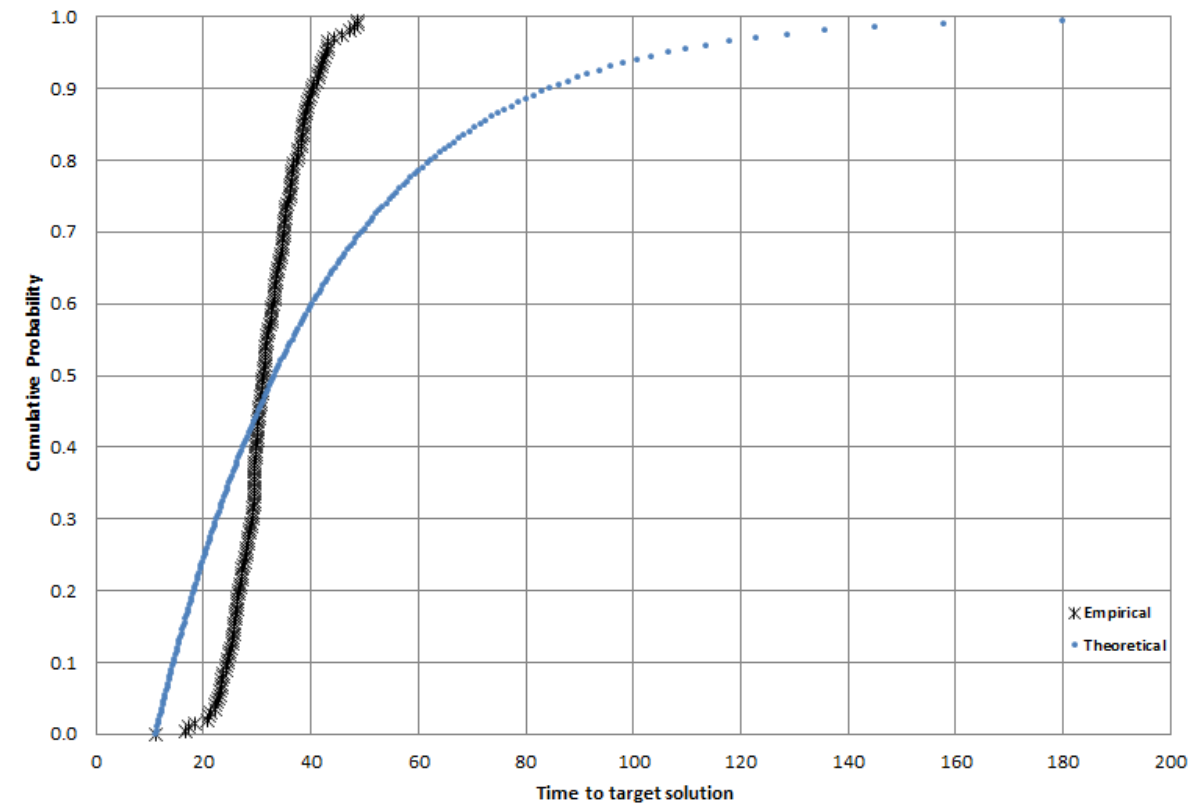

Figure 7: TTT plots produced for instance y.4.80.1 - target value equal to 72 .

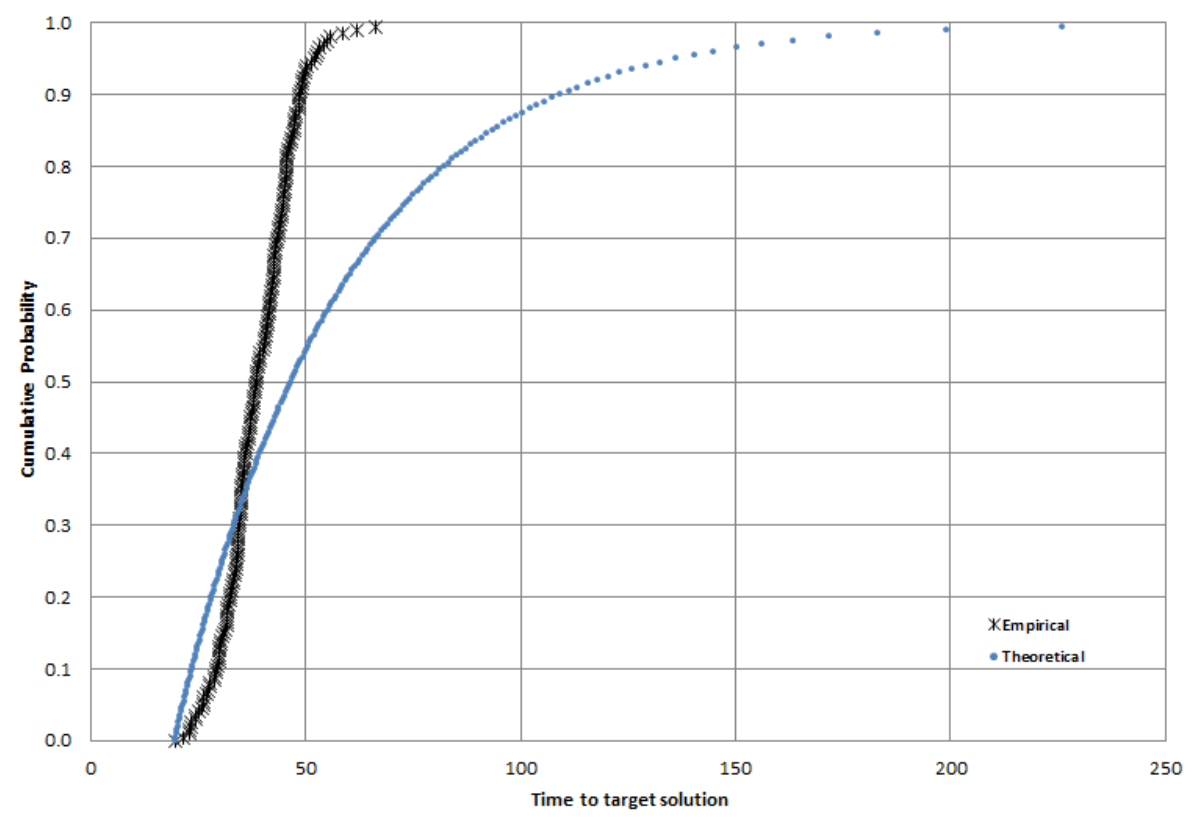

Figure 8: TTT plots produced for instance y.4.100.1 - target value equal to 89. 


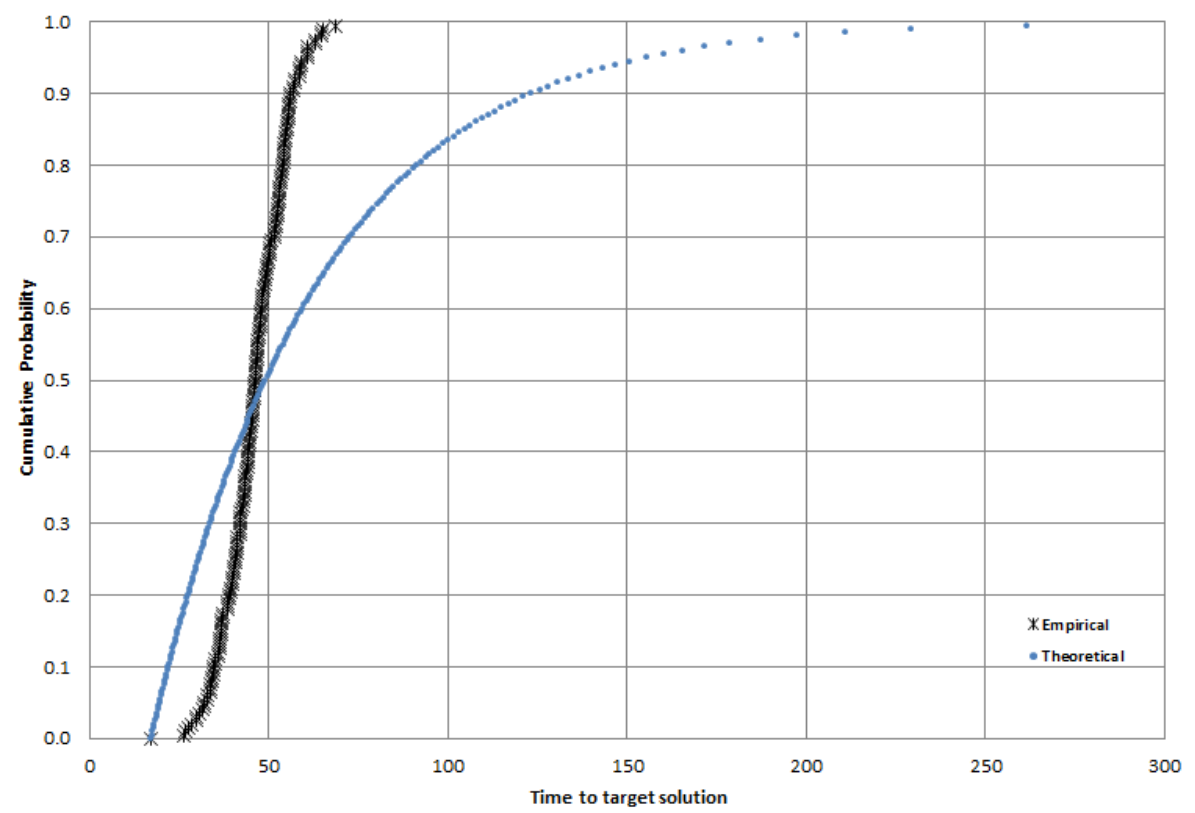

Figure 9: TTT plots produced for instance Z.10x10.60 - target value equal to 86.

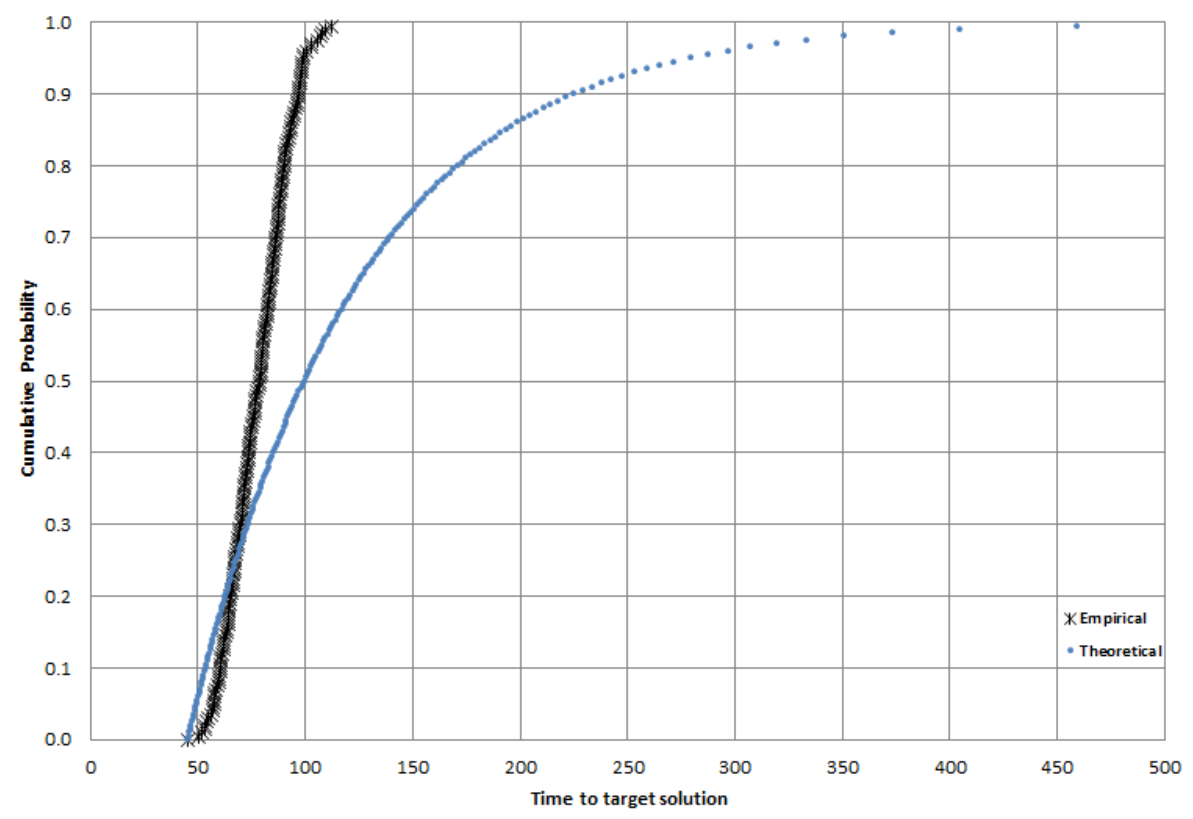

Figure 10: TTT plots produced for instance Z.10x10.80 - target value equal to 114 . 
Multistart-BFD $\quad \mathrm{BFD}^{-\mathrm{VND}_{e}} \quad \mathrm{VND}_{e}-\mathrm{ILS}_{5 p} \quad \mathrm{EDP}_{-\mathrm{VND}} \mathrm{ILS}_{5 p}$

\begin{tabular}{|c|c|c|c|c|c|c|c|c|c|c|c|c|}
\hline Instance & $|V|$ & $|E|$ & $|\Gamma|$ & $\lambda_{\min }$ & $\operatorname{gap}(\%)$ & $\lambda_{\min }$ & $\operatorname{gap}(\%)$ & $\lambda_{\min }$ & $\operatorname{gap}(\%)$ & $\lambda_{\min }$ & $\operatorname{gap}(\%)$ & LB \\
\hline Atlanta20 & 15 & 22 & 13680 & 1342 & 6.91 & 1284 & 2.37 & 1256 & 0.00 & 1256 & 0.00 & 1256 \\
\hline $\mathrm{ATT}$ & 90 & 137 & 359 & 25 & 25.00 & 24 & 20.00 & 20 & 0.00 & 20 & 0.00 & 20 \\
\hline ATT2 & 71 & 175 & 2918 & 113 & 0.71 & 113 & 0.71 & 113 & 0.00 & 113 & 0.00 & 113 \\
\hline Brasil & 27 & 70 & 1370 & 48 & 0.00 & 48 & 0.00 & 48 & 0.00 & 48 & 0.00 & 48 \\
\hline Cost $266^{*}$ & 37 & 57 & 6543 & 446 & 0.00 & 446 & 0.00 & 446 & 0.00 & 446 & 0.00 & 446 \\
\hline Dfn-bwin* & 10 & 45 & 4840 & 73 & 0.00 & 73 & 0.00 & 73 & 0.00 & 73 & 0.00 & 73 \\
\hline Dfn-gwin* & 11 & 47 & 3771 & 316 & 0.00 & 316 & 0.00 & 316 & 0.00 & 316 & 0.00 & 316 \\
\hline $\mathrm{EON}$ & 20 & 39 & 373 & 22 & 0.00 & 22 & 0.00 & 22 & 0.00 & 22 & 0.00 & 22 \\
\hline Finland & 31 & 51 & 930 & 47 & 2.17 & 47 & 2.17 & 46 & 0.00 & 46 & 0.00 & 46 \\
\hline France* & 25 & 45 & 15398 & 946 & 0.00 & 946 & 0.00 & 946 & 0.00 & 946 & 0.00 & 946 \\
\hline Germany50 & 50 & 88 & 4730 & 169 & 15.37 & 169 & 15.37 & 147 & 0.00 & 147 & 0.00 & 147 \\
\hline Giul & 39 & 86 & 14732 & 402 & 6.07 & 401 & 5.91 & 379 & 0.00 & 379 & 0.00 & 379 \\
\hline Janos-us* & 26 & 42 & 3262 & 215 & 4.15 & 215 & 4.15 & 207 & 0.00 & 207 & 0.00 & 207 \\
\hline Nobel-eu & 28 & 41 & 3796 & 304 & 0.00 & 304 & 0.00 & 304 & 0.00 & 304 & 0.00 & 304 \\
\hline Nobel-germany & 17 & 26 & 1320 & 89 & 4.94 & 89 & 4.71 & 85 & 0.00 & 85 & 0.00 & 85 \\
\hline Norway & 27 & 51 & 10696 & 543 & 0.00 & 543 & 0.00 & 543 & 0.00 & 543 & 0.00 & 543 \\
\hline NSF.1 & 14 & 21 & 284 & 23 & 4.55 & 22 & 0.00 & 22 & 0.00 & 22 & 0.00 & 22 \\
\hline NSF.3 & 14 & 21 & 285 & 22 & 3.64 & 22 & 2.73 & 22 & 0.00 & 22 & 0.00 & 22 \\
\hline NSF.12 & 14 & 21 & 551 & 39 & 2.63 & 39 & 2.63 & 38 & 0.00 & 38 & 0.00 & 38 \\
\hline NSF.48 & 14 & 21 & 547 & 41 & 0.49 & 41 & 0.00 & 41 & 0.00 & 41 & 0.00 & 41 \\
\hline NSF2.1 & 14 & 22 & 284 & 21 & 0.00 & 21 & 0.00 & 21 & 0.00 & 21 & 0.00 & 21 \\
\hline NSF2.3 & 14 & 22 & 285 & 21 & 0.00 & 21 & 0.00 & 21 & 0.00 & 21 & 0.00 & 21 \\
\hline NSF2.12 & 14 & 22 & 551 & 35 & 1.71 & 35 & 0.00 & 35 & 0.00 & 35 & 0.00 & 35 \\
\hline NSF2.48 & 14 & 22 & 547 & 39 & 0.00 & 39 & 0.00 & 39 & 0.00 & 39 & 0.00 & 39 \\
\hline Sun & 27 & 51 & 952 & 61 & 3.39 & 60 & 1.69 & 59 & 0.00 & 59 & 0.00 & 59 \\
\hline Average & & & & & 3.27 & & 2.50 & & 0.00 & & 0.00 & \\
\hline
\end{tabular}

Table 2: Comparison with multistart BFD on realistic instances. 


\begin{tabular}{|c|c|c|c|c|c|c|c|c|c|}
\hline \multirow[b]{2}{*}{ Instance } & \multicolumn{2}{|c|}{ Multistart-BFD } & \multicolumn{2}{|c|}{ BFD-VND $e_{e}$} & \multicolumn{2}{|c|}{$\mathrm{VND}_{e}-\mathrm{ILS}_{5 p}$} & \multicolumn{2}{|c|}{$\mathrm{EDP}_{-\mathrm{VND}}-\mathrm{ILS}_{5 p}$} & \multirow[b]{2}{*}{ LB } \\
\hline & $\lambda_{\min }$ & $\operatorname{gap}(\%)$ & $\lambda_{\min }$ & $\operatorname{gap}(\%)$ & $\lambda_{\min }$ & $\operatorname{gap}(\%)$ & $\lambda_{\min }$ & $\operatorname{gap}(\%)$ & \\
\hline y.3.20.1 & 33 & 22.22 & 33 & 22.22 & 29 & 8.89 & 30 & 11.11 & 27 \\
\hline y.3.20.2 & 33 & 0.00 & 33 & 0.00 & 33 & 0.00 & 33 & 0.00 & 33 \\
\hline у. 3.20 .3 & 31 & 6.90 & 31 & 6.90 & 29 & 0.00 & 29 & 0.00 & 29 \\
\hline y.3.20.4 & 31 & 19.23 & 31 & 19.23 & 28 & 8.46 & 29 & 11.54 & 26 \\
\hline у.3.20.5 & 31 & 10.71 & 31 & 10.71 & 28 & 2.86 & 29 & 3.57 & 28 \\
\hline у.3.40.1 & 62 & 18.49 & 62 & 18.11 & 57 & 7.55 & 59 & 11.32 & 53 \\
\hline y.3.40.2 & 59 & 0.00 & 59 & 0.00 & 59 & 0.00 & 59 & 0.00 & 59 \\
\hline y.3.40.3 & 61 & 0.00 & 61 & 0.00 & 61 & 0.00 & 61 & 0.00 & 61 \\
\hline y.3.40.4 & 58 & 16.00 & 58 & 16.00 & 54 & 8.00 & 56 & 12.00 & 50 \\
\hline y.3.40.5 & 60 & 13.21 & 60 & 13.21 & 56 & 6.79 & 58 & 9.43 & 53 \\
\hline y.3.60.1 & 93 & 14.81 & 93 & 14.81 & 87 & 7.41 & 90 & 12.35 & 81 \\
\hline y.3.60.2 & 89 & 0.00 & 89 & 0.00 & 89 & 0.00 & 89 & 0.00 & 89 \\
\hline y.3.60.3 & 91 & 0.00 & 91 & 0.00 & 91 & 0.00 & 91 & 0.00 & 91 \\
\hline y.3.60.4 & 85 & 9.23 & 85 & 8.97 & 80 & 2.82 & 83 & 6.41 & 78 \\
\hline у.3.60.5 & 86 & 12.73 & 86 & 12.73 & 82 & 7.53 & 85 & 11.69 & 77 \\
\hline y.3.80.1 & 123 & 16.04 & 123 & 16.04 & 115 & 9.25 & 122 & 15.09 & 106 \\
\hline y.3.80.2 & 117 & 0.00 & 117 & 0.00 & 117 & 0.00 & 117 & 0.00 & 117 \\
\hline у.3.80.3 & 118 & 0.17 & 118 & 0.17 & 118 & 0.00 & 118 & 0.00 & 118 \\
\hline y.3.80.4 & 112 & 6.67 & 111 & 6.48 & 106 & 1.14 & 110 & 5.71 & 105 \\
\hline у.3.80.5 & 114 & 9.62 & 114 & 9.62 & 109 & 4.81 & 114 & 9.62 & 104 \\
\hline y.3.100.1 & 151 & 15.27 & 151 & 15.57 & 143 & 9.31 & 152 & 16.03 & 131 \\
\hline y.3.100.2 & 146 & 0.00 & 146 & 0.00 & 146 & 0.00 & 146 & 0.00 & 146 \\
\hline y.3.100.3 & 146 & 0.00 & 146 & 0.00 & 146 & 0.00 & 146 & 0.00 & 146 \\
\hline y.3.100.4 & 138 & 5.80 & 138 & 5.80 & 132 & 1.22 & 139 & 6.87 & 131 \\
\hline y.3.100.5 & 141 & 9.30 & 141 & 9.30 & 136 & 5.58 & 143 & 10.85 & 129 \\
\hline Average & & 8.26 & & 8.23 & & 3.66 & & 6.14 & \\
\hline
\end{tabular}

Table 3: Comparison with multistart BFD on set $Y 3$ of instances. 


\begin{tabular}{|c|c|c|c|c|c|c|c|c|c|}
\hline \multirow[b]{2}{*}{ Instance } & \multicolumn{2}{|c|}{ Multistart-BFD } & \multicolumn{2}{|c|}{ BFD-VND $e_{e}$} & \multicolumn{2}{|c|}{$\mathrm{VND}_{e}-\mathrm{ILS}_{5 p}$} & \multicolumn{2}{|c|}{$\mathrm{EDP}_{-\mathrm{VND}}-\mathrm{ILS}_{5 p}$} & \multirow[b]{2}{*}{ LB } \\
\hline & $\lambda_{\min }$ & $\operatorname{gap}(\%)$ & $\lambda_{\min }$ & $\operatorname{gap}(\%)$ & $\lambda_{\min }$ & $\operatorname{gap}(\%)$ & $\lambda_{\min }$ & $\operatorname{gap}(\%)$ & \\
\hline $\mathrm{y} .4 .20 .1$ & 21 & 23.53 & 21 & 23.53 & 19 & 11.76 & 19 & 11.76 & 17 \\
\hline y.4.20.2 & 28 & 0.00 & 28 & 0.00 & 28 & 0.00 & 28 & 0.00 & 28 \\
\hline y.4.20.3 & 23 & 0.00 & 23 & 0.00 & 23 & 0.00 & 23 & 0.00 & 23 \\
\hline y.4.20.4 & 20 & 8.42 & 20 & 7.37 & 19 & 0.00 & 19 & 0.00 & 19 \\
\hline y.4.20.5 & 21 & 23.53 & 21 & 23.53 & 19 & 11.76 & 19 & 15.29 & 17 \\
\hline y.4.40.1 & 38 & 22.58 & 38 & 22.58 & 35 & 12.90 & 36 & 16.13 & 31 \\
\hline y.4.40.2 & 57 & 0.00 & 57 & 0.00 & 57 & 0.00 & 57 & 0.00 & 57 \\
\hline у. 4.40 .3 & 43 & 0.00 & 43 & 0.00 & 43 & 0.00 & 43 & 0.00 & 43 \\
\hline y.4.40.4 & 38 & 0.00 & 38 & 0.00 & 38 & 0.00 & 38 & 0.00 & 38 \\
\hline y.4.40.5 & 40 & 21.21 & 40 & 21.21 & 37 & 12.12 & 38 & 15.15 & 33 \\
\hline y.4.60.1 & 56 & 19.15 & 56 & 19.15 & 53 & 12.77 & 54 & 14.89 & 47 \\
\hline y.4.60.2 & 86 & 0.00 & 86 & 0.00 & 86 & 0.00 & 86 & 0.00 & 86 \\
\hline y.4.60.3 & 64 & 0.00 & 64 & 0.00 & 64 & 0.00 & 64 & 0.00 & 64 \\
\hline y.4.60.4 & 58 & 0.00 & 58 & 0.00 & 58 & 0.00 & 58 & 0.00 & 58 \\
\hline у.4.60.5 & 58 & 19.18 & 58 & 18.78 & 55 & 12.24 & 57 & 16.33 & 49 \\
\hline y.4.80.1 & 73 & 57.02 & 73 & 56.60 & 70 & 48.94 & 72 & 54.04 & 47 \\
\hline $\mathrm{y} \cdot 4.80 .2$ & 118 & 0.00 & 118 & 0.00 & 118 & 0.00 & 118 & 0.00 & 118 \\
\hline y.4.80.3 & 81 & 0.00 & 81 & 0.00 & 81 & 0.00 & 81 & 0.00 & 81 \\
\hline y.4.80.4 & 78 & 0.00 & 78 & 0.00 & 78 & 0.00 & 78 & 0.00 & 78 \\
\hline y.4.80.5 & 76 & 16.92 & 76 & 16.92 & 72 & 10.77 & 75 & 15.38 & 65 \\
\hline y.4.100.1 & 91 & 19.74 & 91 & 19.74 & 86 & 14.21 & 90 & 19.47 & 76 \\
\hline y.4.100.2 & 146 & 0.00 & 146 & 0.00 & 146 & 0.00 & 146 & 0.00 & 146 \\
\hline y.4.100.3 & 98 & 0.00 & 98 & 0.00 & 98 & 0.00 & 98 & 0.00 & 98 \\
\hline y.4.100.4 & 98 & 0.00 & 98 & 0.00 & 98 & 0.00 & 98 & 0.00 & 98 \\
\hline y.4.100.5 & 93 & 16.50 & 93 & 16.50 & 89 & 11.75 & 93 & 17.25 & 80 \\
\hline Average & & 9.91 & & 9.84 & & 6.37 & & 7.83 & \\
\hline
\end{tabular}

Table 4: Comparison with multistart BFD on set $Y 4$ of instances. 


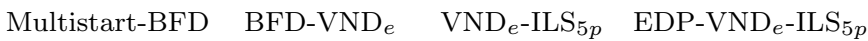

\begin{tabular}{lrrrrrrrrr} 
Instance & $\lambda_{\min }$ & gap $(\%)$ & $\lambda_{\min }$ & gap $(\%)$ & $\lambda_{\min }$ & gap $(\%)$ & $\lambda_{\min }$ & gap $(\%)$ & $\mathrm{LB}$ \\
\hline y.5.20.1 & 14 & 7.69 & 14 & 7.69 & 13 & 0.00 & 13 & 0.00 & 13 \\
y.5.20.2 & 17 & 0.00 & 17 & 0.00 & 17 & 0.00 & 17 & 0.00 & 17 \\
y.5.20.3 & 13 & 8.33 & 13 & 8.33 & 12 & 5.00 & 13 & 8.33 & 12 \\
y.5.20.4 & 17 & 0.00 & 17 & 0.00 & 17 & 0.00 & 17 & 0.00 & 17 \\
y.5.20.5 & 15 & 0.00 & 15 & 0.00 & 15 & 0.00 & 15 & 0.00 & 15 \\
y.5.40.1 & 25 & 4.17 & 25 & 4.17 & 24 & 0.00 & 24 & 0.83 & 24 \\
y.5.40.2 & 31 & 1.29 & 31 & 1.29 & 31 & 0.00 & 31 & 0.00 & 31 \\
y.5.40.3 & 24 & 9.09 & 24 & 9.09 & 23 & 4.55 & 23 & 6.36 & 22 \\
y.5.40.4 & 33 & 0.00 & 33 & 0.00 & 33 & 0.00 & 33 & 0.00 & 33 \\
y.5.40.5 & 28 & 0.00 & 28 & 0.00 & 28 & 0.00 & 28 & 0.00 & 28 \\
y.5.60.1 & 36 & 11.52 & 36 & 11.52 & 35 & 6.67 & 36 & 9.09 & 33 \\
y.5.60.2 & 45 & 1.33 & 45 & 0.89 & 45 & 0.00 & 45 & 0.00 & 45 \\
y.5.60.3 & 35 & 2.94 & 35 & 2.94 & 34 & 0.00 & 34 & 0.00 & 34 \\
y.5.60.4 & 48 & 0.00 & 48 & 0.00 & 48 & 0.00 & 48 & 0.00 & 48 \\
y.5.60.5 & 40 & 0.00 & 40 & 0.00 & 40 & 0.00 & 40 & 0.00 & 40 \\
y.5.80.1 & 47 & 11.16 & 47 & 11.16 & 46 & 6.98 & 47 & 9.30 & 43 \\
y.5.80.2 & 60 & 1.69 & 60 & 1.69 & 59 & 0.00 & 59 & 1.02 & 59 \\
y.5.80.3 & 45 & 4.65 & 45 & 4.65 & 44 & 2.33 & 45 & 5.12 & 43 \\
y.5.80.4 & 63 & 0.00 & 63 & 0.00 & 63 & 0.00 & 63 & 0.00 & 63 \\
y.5.80.5 & 53 & 0.00 & 53 & 0.00 & 53 & 0.00 & 53 & 0.00 & 53 \\
y.5.100.1 & 59 & 7.27 & 59 & 7.27 & 57 & 3.64 & 58 & 5.82 & 55 \\
y.5.100.2 & 75 & 2.74 & 75 & 2.74 & 73 & 0.00 & 74 & 1.37 & 73 \\
y.5.100.3 & 56 & 5.66 & 56 & 5.66 & 54 & 3.40 & 56 & 5.66 & 53 \\
y.5.100.4 & 77 & 0.00 & 77 & 0.00 & 77 & 0.00 & 77 & 0.00 & 77 \\
y.5.100.5 & 66 & 0.00 & 66 & 0.00 & 66 & 0.00 & 66 & 0.00 & 66 \\
\hline Average & & 3.18 & & 3.16 & & 1.30 & & 2.12 & \\
\hline & & & & & & & & &
\end{tabular}

Table 5: Comparison with multistart BFD on set $Y 5$ of instances. 


\begin{tabular}{|c|c|c|c|c|c|c|c|c|c|}
\hline \multirow[b]{2}{*}{ Instance } & \multicolumn{2}{|c|}{ Multistart-BFD } & \multicolumn{2}{|c|}{ BFD-VND $e$} & \multicolumn{2}{|c|}{$\mathrm{VND}_{e}-\mathrm{ILS}_{5 p}$} & \multicolumn{2}{|c|}{$\mathrm{EDP}_{-V N D}-\mathrm{ILS}_{5 p}$} & \multirow[b]{2}{*}{ LB } \\
\hline & $\lambda_{\min }$ & $\operatorname{gap}(\%)$ & $\lambda_{\min }$ & $\operatorname{gap}(\%)$ & $\lambda_{\min }$ & $\operatorname{gap}(\%)$ & $\lambda_{\min }$ & $\operatorname{gap}(\%)$ & \\
\hline $\mathrm{Z} .10 \times 10.20$ & 31 & 17.04 & 31 & 17.04 & 29 & 7.41 & 30 & 11.11 & 27 \\
\hline Z.8x13.20 & 35 & 8.48 & 35 & 8.48 & 34 & 3.03 & 34 & 3.03 & 33 \\
\hline Z. $6 \times 17.20$ & 46 & 4.55 & 46 & 4.55 & 44 & 1.36 & 45 & 2.27 & 44 \\
\hline Z.5x20.20 & 55 & 1.85 & 55 & 1.85 & 54 & 0.00 & 54 & 0.00 & 54 \\
\hline Z. $4 \times 25.20$ & 68 & 3.03 & 68 & 3.03 & 66 & 0.00 & 67 & 2.12 & 66 \\
\hline $\mathrm{Z} .10 \times 10.40$ & 59 & 15.69 & 58 & 14.51 & 55 & 8.63 & 57 & 12.16 & 51 \\
\hline Z.8x13.40 & 67 & 6.35 & 67 & 6.35 & 64 & 2.54 & 66 & 4.76 & 63 \\
\hline Z.6x17.40 & 87 & 4.29 & 87 & 3.57 & 85 & 1.19 & 86 & 2.38 & 84 \\
\hline Z. $5 \times 20.40$ & 104 & 2.97 & 104 & 2.97 & 101 & 0.59 & 103 & 2.18 & 101 \\
\hline $\mathrm{Z} .4 \times 25.40$ & 129 & 2.70 & 129 & 2.38 & 127 & 0.79 & 129 & 2.38 & 126 \\
\hline Z.10x10.60 & 88 & 14.29 & 87 & 14.03 & 84 & 9.09 & 87 & 12.99 & 77 \\
\hline Z.8x13.60 & 101 & 5.63 & 101 & 5.21 & 98 & 2.08 & 100 & 4.38 & 96 \\
\hline Z. $6 \times 17.60$ & 133 & 3.91 & 133 & 3.91 & 129 & 0.78 & 131 & 2.66 & 128 \\
\hline Z.5x20.60 & 158 & 2.60 & 158 & 2.60 & 154 & 0.26 & 158 & 2.60 & 154 \\
\hline Z.4x25.60 & 195 & 2.08 & 195 & 1.98 & 193 & 0.52 & 197 & 2.71 & 192 \\
\hline Z.10x10.80 & 116 & 12.62 & 116 & 12.62 & 112 & 9.51 & 117 & 13.98 & 103 \\
\hline Z.8x13.80 & 134 & 4.50 & 134 & 4.50 & 130 & 1.09 & 134 & 3.88 & 129 \\
\hline Z.6x17.80 & 176 & 3.39 & 176 & 3.27 & 171 & 0.47 & 175 & 2.57 & 171 \\
\hline Z.5x20.80 & 209 & 1.95 & 209 & 1.95 & 206 & 0.49 & 210 & 2.63 & 205 \\
\hline Z.4x25.80 & 261 & 1.56 & 261 & 1.56 & 258 & 0.47 & 264 & 2.72 & 257 \\
\hline Z.10x10.100 & 142 & 13.60 & 142 & 13.60 & 139 & 11.52 & 146 & 17.44 & 125 \\
\hline Z.8x13.100 & 175 & 4.17 & 175 & 4.17 & 173 & 3.21 & 178 & 6.31 & 168 \\
\hline Z.6x17.100 & 222 & 3.06 & 222 & 3.06 & 220 & 1.85 & 225 & 4.17 & 216 \\
\hline Z.5x20.100 & 256 & 2.56 & 256 & 2.48 & 253 & 1.52 & 260 & 4.16 & 250 \\
\hline Z.4x25.100 & 319 & 2.24 & 318 & 2.18 & 317 & 1.60 & 325 & 4.42 & 312 \\
\hline Average & & 5.80 & & 5.67 & & 2.80 & & 5.20 & \\
\hline
\end{tabular}

Table 6: Comparison with multistart BFD on set $Z$ of instances. 


\begin{tabular}{lrrrrr} 
& \multicolumn{1}{c}{ GA-RWA } & \multicolumn{3}{c}{ VND $_{e}$-ILS $5 p$} \\
Instance & $\lambda_{\text {min }}$ & gap $\%$ ) & $\lambda_{\text {min }}$ & gap $(\%)$ & LB \\
\hline ATT & 24 & 20.0 & 20 & 0.0 & 20 \\
ATT2 & 113 & 0.0 & 113 & 0.0 & 113 \\
Finland & 46 & 0.4 & 46 & 0.0 & 46 \\
NSF.3 & 22 & 0.9 & 22 & 0.0 & 22 \\
NSF.12 & 39 & 2.6 & 38 & 0.0 & 38 \\
NSF2.12 & 35 & 0.6 & 35 & 0.0 & 35 \\
Z.10x10.20 & 31 & 15.6 & 29 & 7.4 & 27 \\
Z.6x17.40 & 87 & 4.0 & 85 & 1.2 & 84 \\
Z.10x10.60 & 87 & 13.2 & 84 & 9.1 & 77 \\
Z.4x25.60 & 195 & 2.0 & 193 & 0.5 & 192 \\
Z.10x10.80 & 115 & 12.4 & 112 & 9.5 & 103 \\
Z.8x13.80 & 134 & 3.9 & 130 & 1.1 & 129 \\
Z.6x17.80 & 176 & 3.0 & 171 & 0.5 & 171 \\
Z.5x20.80 & 209 & 2.0 & 206 & 0.5 & 205 \\
Z.4x25.80 & 260 & 1.3 & 258 & 0.5 & 257 \\
Z.5x20.100 & 257 & 2.8 & 253 & 1.5 & 250 \\
y.4.20.4 & 20 & 6.3 & 19 & 0.0 & 19 \\
y.3.40.5 & 59 & 12.8 & 56 & 7.4 & 53 \\
y.3.60.5 & 86 & 12.5 & 82 & 7.5 & 77 \\
y.4.60.5 & 58 & 18.4 & 55 & 12.2 & 49 \\
y.5.60.1 & 36 & 9.7 & 35 & 6.7 & 33 \\
y.3.80.1 & 122 & 15.5 & 115 & 9.3 & 106 \\
y.3.80.5 & 113 & 8.8 & 109 & 4.8 & 104 \\
y.4.80.1 & 73 & 55.3 & 70 & 48.9 & 47 \\
y.4.80.5 & 75 & 16.0 & 72 & 10.8 & 65 \\
y.5.80.1 & 47 & 11.2 & 46 & 7.0 & 43 \\
y.5.80.2 & 60 & 1.7 & 59 & 0.0 & 59 \\
y.4.100.1 & 90 & 18.4 & 86 & 14.2 & 76 \\
y.5.100.1 & 58 & 5.5 & 57 & 3.6 & 55 \\
y.5.100.2 & 74 & 1.6 & 73 & 0.0 & 73 \\
\hline Average & & 9.3 & & 5.5 & \\
\hline & & & & &
\end{tabular}

Table 7: Comparison with GA-RWA on a set of difficult instances. 


\begin{tabular}{|c|c|c|c|c|c|c|c|c|c|c|c|}
\hline \multirow[b]{2}{*}{ Instance } & \multicolumn{2}{|c|}{$N_{1}$} & \multicolumn{2}{|c|}{$N_{2}$} & \multicolumn{2}{|c|}{$N_{1}+N_{2}$} & \multicolumn{2}{|c|}{$N_{1}+N_{3}$} & \multicolumn{2}{|c|}{$N_{2}+N_{3}$} & \multirow[b]{2}{*}{ LI } \\
\hline & $\lambda_{\min }$ & $\operatorname{gap}(\%)$ & $\lambda_{\min }$ & $\operatorname{gap}(\%)$ & $\lambda_{\min }$ & $\operatorname{gap}(\%)$ & $\lambda_{\min }$ & $\operatorname{gap}(\%)$ & $\lambda_{\min }$ & $\operatorname{gap}(\%)$ & \\
\hline ATT & 20 & 0.0 & 26 & 33.3 & 20 & 0.0 & 20 & 0.0 & 20 & 0.0 & 20 \\
\hline ATT2 & 113 & 0.0 & 115 & 2.0 & 113 & 0.0 & 113 & 0.0 & 113 & 0.0 & 113 \\
\hline Finland & 46 & 0.0 & 48 & 5.2 & 46 & 0.0 & 46 & 0.0 & 46 & 0.0 & 46 \\
\hline NSF.3 & 22 & 0.0 & 22 & 0.0 & 22 & 0.0 & 22 & 0.0 & 22 & 0.0 & 22 \\
\hline NSF.12 & 38 & 0.0 & 38 & 0.0 & 38 & 0.0 & 38 & 0.0 & 38 & 0.0 & 38 \\
\hline NSF2.12 & 35 & 0.0 & 35 & 0.0 & 35 & 0.0 & 35 & 0.0 & 35 & 0.0 & 35 \\
\hline Z.10x10.20 & 30 & 14.1 & 32 & 20.7 & 30 & 11.1 & 29 & 7.4 & 29 & 7.4 & 27 \\
\hline Z.6x17.40 & 87 & 3.6 & 88 & 5.5 & 85 & 2.1 & 86 & 2.4 & 85 & 1.2 & 84 \\
\hline Z.10x10.60 & 88 & 14.3 & 89 & 15.8 & 86 & 12.7 & 85 & 10.4 & 85 & 10.4 & 77 \\
\hline Z.4x25.60 & 195 & 2.2 & 196 & 3.1 & 193 & 1.0 & 195 & 1.7 & 193 & 0.9 & 192 \\
\hline Z.10x10.80 & 115 & 12.2 & 116 & 13.6 & 115 & 11.8 & 114 & 10.7 & 113 & 9.7 & 103 \\
\hline Z.8x13.80 & 134 & 3.9 & 135 & 5.0 & 132 & 2.9 & 131 & 1.9 & 131 & 1.6 & 129 \\
\hline Z. $6 \times 17.80$ & 175 & 2.7 & 177 & 4.0 & 173 & 1.3 & 173 & 1.2 & 172 & 0.7 & 171 \\
\hline Z.5x20.80 & 209 & 2.1 & 210 & 2.8 & 206 & 0.9 & 207 & 1.4 & 206 & 0.6 & 205 \\
\hline Z.4x25.80 & 260 & & 262 & & 259 & 8 & 260 & 1.3 & 259 & 0.8 & 257 \\
\hline Z.5x20.100 & 256 & 2.5 & 257 & 3.0 & 254 & 1.9 & 255 & 2.3 & 254 & 1.7 & 250 \\
\hline у.4.20.4 & 19 & 0.0 & 21 & 11.6 & 19 & 4.2 & 19 & 0.0 & 19 & 0.0 & 19 \\
\hline у.3.40.5 & 58 & 10.9 & 60 & 14.7 & 58 & 9.4 & 57 & 7.5 & 57 & 7.5 & 53 \\
\hline у.3.60.5 & 85 & 11.4 & 87 & 13.5 & 84 & 10.1 & 83 & 8.3 & 83 & 7.8 & 77 \\
\hline у.4.60.5 & 58 & 18.4 & 59 & 21.2 & 58 & 18.4 & 55 & 13.5 & 55 & 12.2 & 49 \\
\hline у.5.60.1 & 36 & 10.9 & 37 & 14.5 & 36 & 10.3 & 35 & 6.7 & 35 & 8.5 & 33 \\
\hline у.3.80.1 & 121 & 14.5 & 123 & 17.2 & 119 & 12.8 & 116 & 10.0 & 116 & 9.6 & 106 \\
\hline у.3.80.5 & 113 & 8.7 & 114 & 10.0 & 111 & 7.5 & 110 & 5.8 & 109 & 5.4 & 104 \\
\hline y.4.80.1 & 73 & 55.7 & 74 & 58.3 & 73 & 55.3 & 70 & 49.4 & 70 & 49.4 & 47 \\
\hline y.4.80.5 & 75 & 15.7 & 76 & 17.8 & 75 & 15.4 & 73 & 12.3 & 72 & 11.7 & 65 \\
\hline у. 5.80 .1 & 47 & 11.2 & 49 & 14.0 & 47 & 11.2 & 46 & 7.0 & 46 & 7.9 & 43 \\
\hline y.5.80.2 & 59 & 1.4 & 60 & 2.7 & 59 & 1.0 & 59 & 0.0 & 59 & 0.0 & 59 \\
\hline y.4.100.1 & 90 & 18.9 & 91 & 20.3 & 90 & 18.7 & 87 & 14.5 & 87 & 14.5 & 76 \\
\hline y.5.100.1 & 58 & 5.5 & 59 & 8.4 & 58 & 5.5 & 57 & 3.6 & 57 & 3.6 & 55 \\
\hline y.5.100.2 & 74 & 1.4 & 76 & 4.1 & 74 & 1.4 & 73 & 0.5 & 73 & 0.5 & 73 \\
\hline Average & & 8.1 & & 11.5 & & 7.6 & & 6.0 & & 5.8 & \\
\hline
\end{tabular}

Table 8: Analysis on the performance of each neighborhood. 